Running head: Eyes to hand... hand to eyes?

\section{EFFECTS OF WRIST TENDON VIBRATION AND EYE MOVEMENTS ON MANUAL AIMING}

9

)

1

2

13

Please address all correspondence to:
Ann Lavrysen
Movement Control and Neuroplasticity
KU Leuven - Department of Movement Sciences
Tervuursevest 101, box 1501
B-3001 Leuven (Belgium)
Phone: $\quad+32-16-37.95 .98$
Fax: $\quad+32-16-32.91 .97$
E-mail: ann.lavrysen@kuleuven.be 


\section{Abstract}

In the present study, we investigated whether visual information mediates a proprioceptive illusion effect induced by muscle tendon vibration in manual aiming. Visual information was gradually degraded from a situation in which the targets were present and participants $(n=20$; $22.3 \pm 2.7 \mathrm{yrs})$ were permitted to make saccadic eye movements to designated target positions, to a condition in which the targets were not visible and participants were required to perform cyclical aiming while fixating a point between the two target positions. Local tendon vibration applied to the right wrist extensor muscles induced an illusory reduction of $15 \%$ in hand movement amplitude. This effect was greater in the fixation than in the saccade condition. Both anticipatory control and proprioceptive feedback are proposed to contribute to the observed effects. The primary saccade amplitude was also reduced by almost $4 \%$ when muscle tendon vibration was locally applied to the wrist. These results confirm a tight link between eye movements and manual perception and action. Moreover, the impact of the proprioceptive illusion on the ocular system indicates that the interaction between systems is bidirectional.

Keywords: tendon vibration; eye-hand coordination; perceptual illusion; sensory information 


\section{INTRODUCTION}

There are close links and interactions between $\underline{P}-$ perception and action processes are closely linked in goal-directed movements. Evidence that eye and hand movements influence each other and that visual information is being shared by both effector systems comes from studies examining eye-hand coordination in perceptual illusion paradigms. For example, hand and eye movements have been shown to be biased by visual illusions when aiming to MüllerLyer images (Lavrysen et al, 2006). The goal of the present study is-was to investigate the impact of a proprioceptive illusion caused by tendon vibration applied to the wrist on the planning of hand and eye movements. To the best of our knowledge, it has notever been demonstrated that hand movement bias caused by proprioceptive illusion influences eye movements.

In goal-directed tasks, sensory information is continuously being updated and integrated into the motor plan to ensure successful execution. Three main sources of visual and proprioceptive feedback are used. The combined signal from retinal and extraretinal sources provides integrated position and movement information (Binsted \& Elliott, 1999). Proprioceptive information coming from the muscle spindles in the arm is used to determine the position of the arm in space (Bekkering \& Sailer, 2002). By independently manipulating these 3 sources of information, one can investigate the interplay and mutual relationships between vision, proprioception and action. If sensory feedback from one source is unpredictable, optimal movement control can be maintained through adjustments in the sensory contributions (e.g., sensory reweighting in postural control; Assländer \& Peterka, 2014). Similarly, the degree of illusory bias on hand movement is determined by the availability of current visual information about the hand and target (Binsted \& Elliott, 1999; Elliott \& Lee, 1995; Lavrysen et al., 2006). Different sensory modalities can thus influence each other. In the current study, the reliability and amount of both proprioceptive and visual information was manipulated to study the relative contribution of, and interplay between, different sensory feedback sources. The aim of the present experiment was to determine to what extent visual non-movement information and muscle tendon vibration entediate illusion effects on eye and hand movements.

Perturbation studies provide valuable information about the processes involved in the visual guidance of movements and the interplay between ocular and manual systems. Visual illusions like the Müller-Lyer (ML) illusion have been studied frequently in discrete aiming tasks (Binsted \& Elliott, 1999; Binsted, Chua, Helsen, \& Elliott, 2001; van Donkelaar, 1999). 
In these studies, the participants typically make horizontal aiming movements with the hand to the vertex of an inward or outward pointing Müller-Lyer figure and a control figure. The effect of the illusion on hand and/or eye movements is measured. The common finding is that saccades typically undershoot the outward aiming figures while they overshoot, or undershoot to a lesser extent, the inward pointing version (Bruno, Knox, \& de Grave, 2010). Hand movements seem to be less affected by the illusion (Bruno, Bernardis, \& Gentilucci, 2008). These results have been used to posit a dichotomy between vision for perception and vision for action (Goodale \& Milner 1992, Milner \& Goodale, 1995; Franz \& Genenfurtner, 2008).

The magnitude of visual illusion effects on eye and hand movements and their coordination is mediated by the amount and precision of visual information, and the possibility for comparison between hand and target information (Mendoza, Elliott, Meegan, Lyons, \& Welsh, 2006). Binsted and colleagues (1999) for instance showed that making concurrent eye and hand movements, and the absence of retinal target information were prerequisites for an illusory influence. Also Lavrysen and colleagues (2006) reported that ML illusion effects on both eye and hand movements were mediated by the amount and type of visual feedback (see also Gentilucci, Chieffi, Daprati, Saetti, \& Toni, 1996). Contrary to Binsted and colleagues (1999), the illusion was not greater when making eye-hand coordinated movements, than when making only hand movements (fixation condition). Nonetheless, participants did show less endpoint variability when making saccades as compared to fixating. Although often disregarded, saccades have a beneficial effect on aiming accuracy as they permit the online guidance and updating of retinal and non-retinal information about limb and/or target and position in space.

The above mentioned sStudies involving both discrete and reciprocal aiming show that saccades have a beneficial effect on performance and potentially moderate illusory bias. Although there is plenty of evidence that saccades impact hand movements, there are also data indicating that arm proprioception affects the ocular system (Lunenburger, Kutz, \& Hoffmann, 2000; Tipper, Howard, \& Paul, 2001). In the context of pursuit tracking, the concurrent performance of eye and hand movements benefits smooth pursuit eye movements over isolated action (Gauthier, Vercher, Mussa Ivaldi, \& Marchetti, 1988). In manual aiming, the interaction from hand to eye movements is believed to result from an efference copy of the motor command. This feedforward source of information provides more specific kinetic and kinematic information about the hand movement, than prioprioception (van Donkelaar, Siu, \& Walterschied, 2004; Vercher et al., 1996). Together, these studies suggest that eye movements have beneficial effects on hand movements, which includes reducing the impact of illusory 
bias. Therefore, visual information and eye movements should not be ignored when considering illusory effects on goal-directed movements.

The effects of proprioceptive perturbations such as tendon vibration (TV) have been investigated to a far lesser extent than visual perturbations. Typically, vibrating an agonistic muscle of a moving limb evokes a kinaesthetic illusion towards the antagonistic direction and thus leads to a target undershoot (Kasai, Kawanishi, \& Yahagi, 1992, 1994; Verschueren, Cordo, \& Swinnen, 1998). The effects of vibration vary with factors such as frequency or timing/onset of vibration (Cordo, Gurfinkel, Bevan, \& Kerr, 1995), but the possible mediating impact of vision on the TV illusion has been neglected. Tendon vibration (TV) experiments are typically done with eyes closed or at least with no vision of the ongoing movement, in order to isolate the proprioceptive effect. A rare exception where eye movements were measured, is a study by Tardy-Gervet and colleagues (1989) in which participants were asked to fixate their hand in complete darkness while their arm was isometrically vibrated. Their results showed that perturbed proprioceptive information from the arm also affected or at least interacted with the oculomotor system. Specifically, ocular movements were elicited either in the same, or in the opposite direction of the arm movements.

Despite the large body of literature on visual illusions, studies have been unable to determine whether the observed effects on eye and hand movements are due to a common command (i.e., feedforward information available prior to the movement) or because of crosstalk between information sources during the course of the movement (Franz, Gegenfurtner, Bülthoff, \& Fahle, 2000; Milner \& Goodale, 2008). Thus, the question remains: Are eye and hand movements being programmed based on a common source of information (van Donkelaar, 1999) or do they rely on different target representations that interconnect (Bekkering \& Sailer, 2002; Lazzari, Vercher, \& Buizza, 1997)? In this theoretical context, there might be cross-talk at the level of programming of the two effectors, showing that, to some extent, they operate based on separate but shared information sources. In case of the ML illusion, the target figure is typically presented before movement onset. This protocol allows participants to make a judgement about the distance-to-travel. This judgement could affect the planning of both eye and hand movements. Suboptimal planning could then be corrected during movement execution. This would explain the differential online effects on the two effectors, during movement execution. Thus, both prior knowledge and online updating of the trajectory could explain the observed effects. In contrast to visual illusions, muscle TV introduces a local effect on the hand that is not always noticed. Nevertheless, recent studies reveal that tendon vibration not only activates the local muscle spindles, but also evokes brain activity (Naito, 2005; Goble 
et al., 2011), specifically in the right inferior frontoparietal network (Cignetti et al. 2014). Some of the observed activation has been specifically attributed to perceiving the illusory bias (Casini et al., 2006; Romaiguère et al., 2003). As this type of bias results from sensory (mis)perception, it may not influence the movement planning that occurs prior to movement initiation in discrete movement paradigms. Thus, in a discrete TV paradigm, 1) saccade movement planning is not affected as the participants are not yet aware of the amplitude shortening in advance of the movement, and 2) as the eye movements are finished well before the hand movements, information transfer from eyes to hand is unlikely. The fact that mainly discrete movements have been studied may explain why only local effects of TV on hand movements have been reported and the impact of vision and eye movements has been neglected.

In the present study, we wanted to test the presumed effect of TV on vision. loeal TV was applied during cyclical goal-directed wrist movements. We used cyclical instead of discrete aiming movements first, in order to give the tendon vibration illusion sufficient time to develop (Tidoni et al., 2014) and second, to allow for an interplay between perception and action planning. Our goal was to determine whether the amount of visual information would mediate the illusion evoked by tendon vibration of the wrist. We used cyclical instead of discrete aiming movements, first, in order to give the tendon vibration illusion sufficient time to develop (Tidoni et al., 2014) and second, to allow for an interplay between perception and action planning. Vision was manipulated in two ways. Specifically, participants either performed wrist aiming movements with concurrent eye movements, or while fixating a centre position between the two targets. Our second manipulation involved participants aiming under concurrent target or remembered target conditions. In line with previous studies, a reduction of hand movement amplitude due to TV was expected in all conditions. Furthermore, it was hypothesized that the size of the illusion would be reduced when eye movements were permitted and when the targets remained visible over the course of an aiming trial. Finally, under saccadic eye movement conditions, limb bias was expected to affect eye movements but to a somewhat smaller extent. This latter finding would provide evidence for bi-directional communication between the ocular-motor and manual sensory-motor systems.

\section{MATERIALS AND METHODS}

\subsection{Participants}

Twenty young adults $(22.3 \pm 2.7$ yrs; 8 females $)$ voluntarily participated in the study. All participants were naive regarding the hypotheses. The participants were self-reported righthanders and had normal or corrected-to-normal vision (no glasses due to the eye tracker). They 
had no reported neurological deficits or musculo-skeletal problems at the forearm. Prior to the experiment, written informed consent was obtained from all participants. The study was approved by the KU Leuven Committee for Ethical Considerations in Human Experimentation.

\subsection{Apparatus}

The participants were seated in front of a computer screen positioned on a table. The distance between the eyes and the screen was approximately $1.25 \mathrm{~m}$. A neck support attached to the chair prevented head movements without restricting the head. The right forearm was positioned in an orthosis attached to the armchair with the thumb up (Lavrysen et al., 2006; Van Halewyck et al., 2014). This orthosis allowed wrist flexion/extension movements that were measured with a high-precision optical shaft encoder (Sick ${ }^{\circledR}$ DSF60) with a resolution of 65536 points per rotation $\left(0.0055^{\circ}\right)$ at a sampling rate of $250 \mathrm{~Hz}$. Local tendon vibration was applied using a cylindrical shaker similar to the one used in Steyvers, Verschueren, Levin, Ouamer, and Swinnen (2001). It was positioned approximately $1 \mathrm{~cm}$ proximal to the lateral epicondylus of the wrist on the extensor muscles. The apparatus vibrated transcutaneously at a frequency of 40 $\mathrm{Hz}$ with an amplitude of $1 \mathrm{~mm}$. These settings produced stable vibration effects in all participants in a pilot study. The eye movements were recorded by an Applied Sciences Laboratory pan-tilt eye-tracker (ASL- $6000^{\circledR}$ ) with an accuracy of $0.05^{\circ}$ at a sampling rate of $240 \mathrm{~Hz}$. The data of hand and eye movements were integrated on a stimulus PC.

\subsection{Task}

The test setup is shown in Figure $1 \underline{\mathrm{A} \text { and } \mathrm{B}}$. The participants' task was to perform a series of wrist flexion and extension movements over a range of $28.7^{\circ}$ in time with an auditory pacing signal. A metronome paced 30 movements per trial with a frequency of $1.67 \mathrm{~Hz}$ (ISI $600 \mathrm{~ms}$; duration $50 \mathrm{~ms}$ ). A round cursor of $10 \mathrm{~mm}$ diameter represented the angular position of the hand during the practice trials. On the screen, two black target squares were presented left and right with a distance of $232.4 \mathrm{~mm}$ between the centres of the targets. As movements were made in the horizontal plane, wrist flexion and extension movements corresponded with a movement of the cursor to the left versus right, respectively. Direct vision of the hand was prevented by a wooden panel. An additional benefit of the indirect mapping setup was to allow manipulation of the vision conditions (hand and targets). 

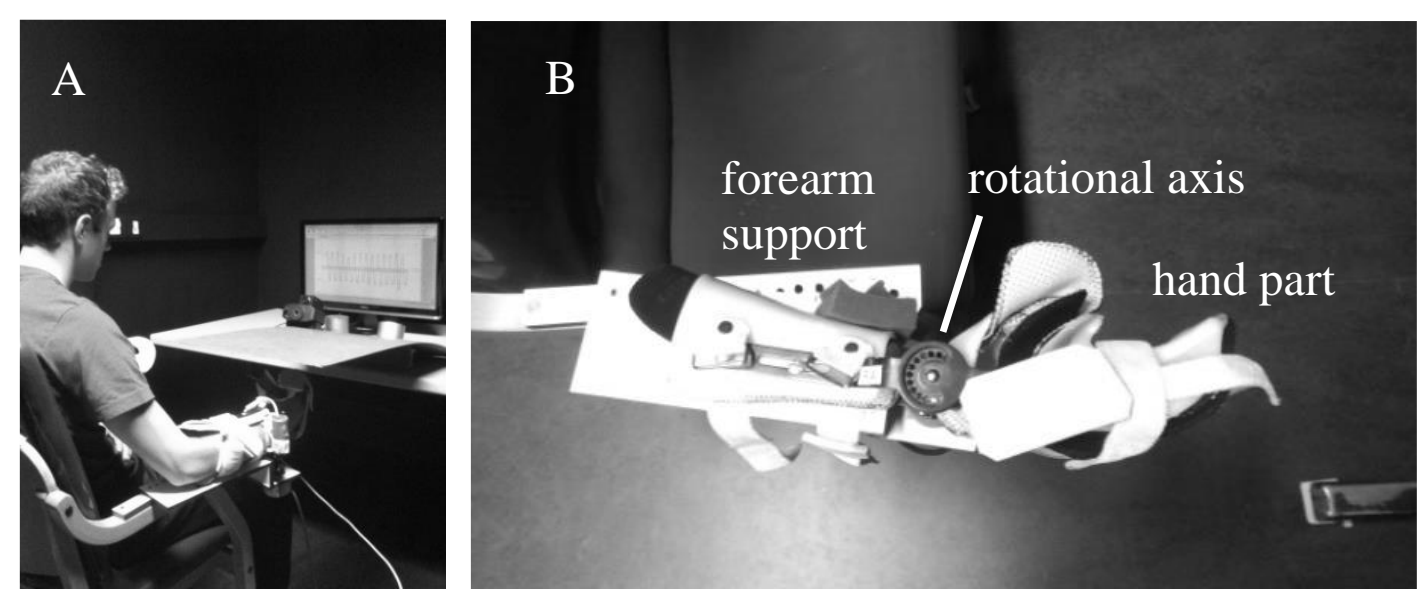

Figure 1: Test setup. (A) Participants were instructed to perform wrist flexionextension movements while looking at a computer screen. Direct vision of their arm was prevented by a wooden panel. Eye movements were recorded by an ASL eye tracker in front of the screen. The right forearm was placed in an orthosis and the shaker was positioned on the wrist extensor tendons. (B) Top view of the orthosis, with the forearm part attached to the arm chair.

During each trial, vision of the cursor disappeared after the tenth beep and the participants were instructed to continue the task for the next 20 beeps as accurately as possible in time with the metronome. Saccades were allowed in half of the trials. In the remaining half, participants were instructed to fixate on an additional target in the middle of the screen (i.e., fixation point) throughout the trial. Additionally, visual information was manipulated by removing visual feedback of the targets in half of the trials after the tenth beep along with the cursor. As such, four conditions were obtained with decreasing visual information: saccades/targets (SAC-T); saccades/no targets (SAC-NT); fixation/targets (FIX-T); fixation/no targets (FIX-NT). All these conditions were performed in a blocked design in pairs of a novibration, followed by a vibration trial. The use of one-dimensional wrist flexion extension movements enabled us to isolate agonist versus antagonist muscle groups for the tendon vibration manipulation. Vibrating the wrist extensor tendons would thus result in a shortening of the movement towards the left/wrist flexion side. 


\subsection{Procedure}

Before the start of the practice trials, the shaker was applied and switched on to acquaint participants with the sensation of the vibration prior to the actual experiment. Then, five practice trials were given in which two were performed with saccades and three in which they were instructed to fixate the additional point between the two targets. These practice trials were a pair of trials with one with full-vision followed by a no-vision trial (30 beeps each). After each practice trial, feedback was provided about the no-vision trial focusing on accuracy and stability of hand movements. Before the actual experiment, the eye-tracker was calibrated using a nine-point calibration.

Each of the four experimental conditions (i.e., SAC-T, SAC-NT, FIX-T, and FIX-NT) was repeated 6 times in blocks of 3 . The order of these blocks was randomized across participants. A short break was given halfway through the experiment, dividing the session into two identical parts with each condition occurring three times. The tasks werewas presented in pairs of two trials (see Fig 2): first the control condition without vibration and-followed by the vibration condition in which the shaker was switched on one beep after the cursor disappeared (i.e., the 11th beep). The remaining "no-vision part" of each trial was used for analysis. break was given halfway through the experiment, dividing the session into identical parts with hation couring three times.

$\underline{\mathrm{A}}$

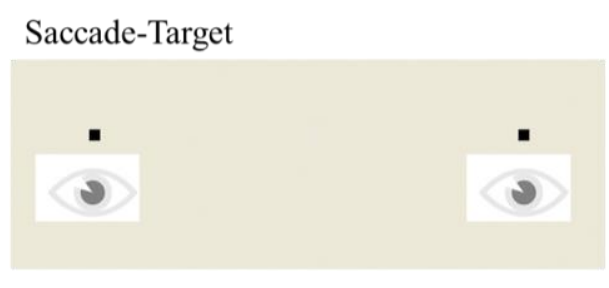

Saccade-NoTarget

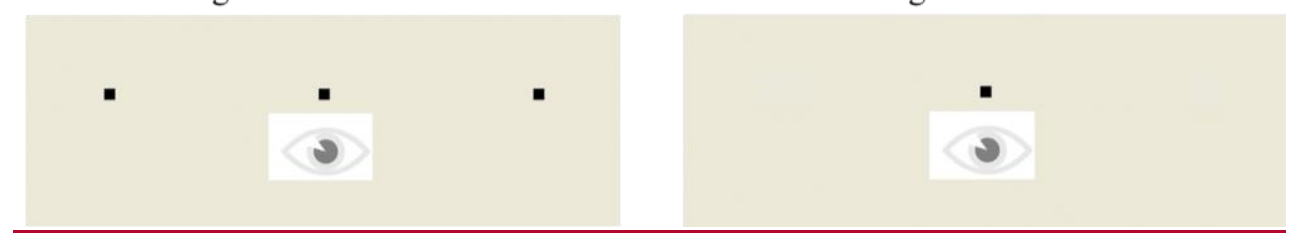

$\underline{B}$ 


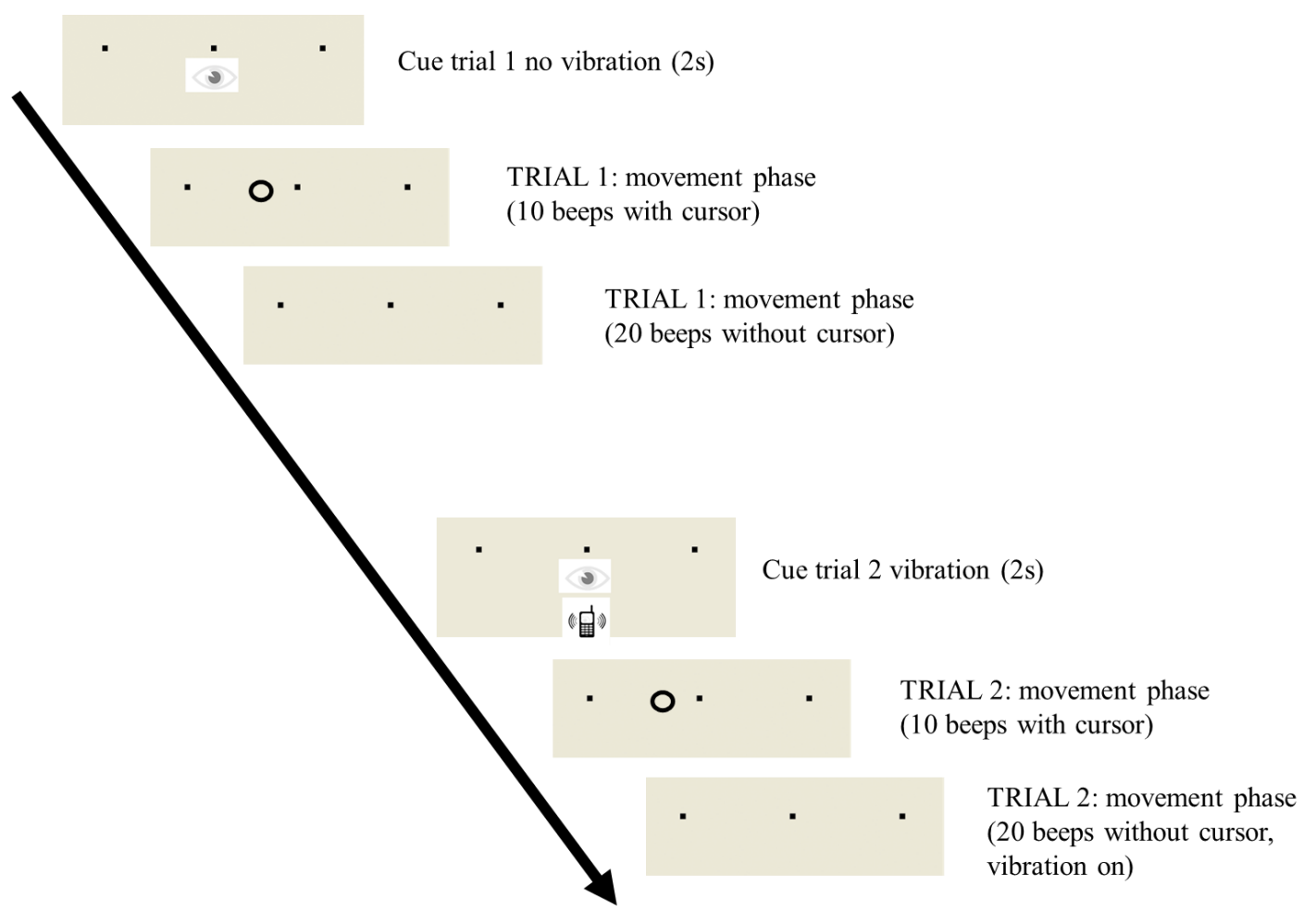

Figure 2: Task presentation. (A) Cues depicting the different conditions, presented before each trial. During the trial, the participants saw the information of target and/or fixation points, but without the eyes. (B) Schematic overview of a Fixation-Target trial block. After short presentation of the visual cue, the first trial started without vibration, immediately followed by the visual cue and trial for the vibration condition. Only movements made without feedback (no cursor) were used for the analyses.

If necessary, the eye calibration procedure was repeated after the break. Thus, each condition was repeated six times in blocks of three in a randomized order.Participants were informed in advance about the upcoming task and were reminded by a visual cue immediately prior to each trial. All participants were naïve to the effects of vibration and none of them reported to have noticed any effects after the experiment. They all thought they were aiming accurately and were surprised about the effects when shown their performance on one of the vibration trials during debriefing after the experiment.

\subsection{Dependent variables}

Hand movements. Raw wrist position data were filtered using a first order low-pass Butterworth filter with a cut-off frequency of $20 \mathrm{~Hz}$ and then differentiated once to obtain the velocity and twice for acceleration. The start of the hand movement was defined as the moment 
in which velocity surpassed $100 \mathrm{~mm} / \mathrm{s}$ and, similarly, the end of the hand movement in which velocity fell below $100 \mathrm{~mm} / \mathrm{s}$. The distance and time between the start and end position was the amplitude (AMPh) and movement time (MTh) of the hand respectively. Furthermore, peak velocity (PV) and the timing of PV relative to the hand movement time (\%TTPV) were calculated.

Eye movements. Similar to the hand movement data, the eye movement data were filtered with a first order low pass Butterworth filter with a cut-off frequency of $20 \mathrm{~Hz}$. The method used to determine saccade initiation and termination was similar to the one used by Helsen et al. (1998a). A standard deviation of 8 consecutive points (i.e., more than $32 \mathrm{~ms}$ ) greater or smaller than $5 \mathrm{~mm}$ on screen marked the start and endpoint of the primary saccade, respectively. Furthermore, the saccade duration had to be longer than $30 \mathrm{~ms}$ but shorter than 150 ms. Only hand movements that were accompanied by a saccade were withheld and the percentage of movements accompanied by saccades was calculated (number of eye movements). The amplitude of the primary saccade (AMPs) was calculated as the distance between the start and endpoint of the first saccade towards the target. These timepoints of the start (StartSac) and endpoint of the saccade (EndSac) were also expressed as a percentage of hand movement time to quantify eye-hand coordination.

\subsection{Design}

For each variable, the mean of all movements per trial was calculated. The first movements after removal of vision / onset of vibration were not analysed. Specifically, on average, 15 movements per trial were used for the analysis ${ }^{1}$. These means were then aggregated over six repetitions to obtain one estimate for each participant in each condition. The dependent variables of the hand were analysed using a 2 VIBRATION (No Vibration, Vibration) $\times 2$ VISION (Saccades, Fixation) $\times 2$ TARGET (No Targets, Targets) repeated measures ANOVAs. The eye movement and eye-hand coordination data were analysed for the Saccade conditions only using 2 VIBRATION (No vibration, Vibration) $\times 2$ TARGET (No Targets, Targets) repeated measures ANOVAs. All statistical tests were completed with alpha set at < 0.05. Results are presented as the group mean and standard error scores.

\subsection{Hand movements}

Hand movement time (MTh). The average hand movement time was $525.6 \pm 11.6 \mathrm{~ms}$. With an ISI of $600 \mathrm{~ms}$, indicating that participants generally obeyed the instructions for 
movement time and had ample time to execute the hand movements. There was a significant effect for TARGET $[F(1,19)=7.805, p<.05]$ with a shorter hand movement time in the No Target $(521.3 \pm 11.5 \mathrm{~ms})$ than in the Target condition $(529.8 \pm 12.4 \mathrm{~ms})$.

Hand amplitude (AMPh). The analysis revealed significant main effects for VIBRATION $[F(1,19)=75.193, p<.001]$, TARGET $[F(1,19)=23.412, p<.001]$, and for VISION $[F(1,19)=8.525, p<.01]$, and interaction effects involving VIBRATION and VISION $[F(1,19)=10.038, p<.01]$, and VISION and TARGET $[F(1,19)=10.442, p<.01]$. The amplitude was smaller when the shaker was switched on (192.2 $\pm 5.5 \mathrm{~mm})$ compared to aiming without vibration $(227 \pm 3.4 \mathrm{~mm})$. The illusory reduction in amplitude thus averaged $15.3 \%$. The amplitude was overall smaller in the Saccade $(205.3 \pm 3.3 \mathrm{~mm})$ than in the Fixation condition $(213.9 \pm 5.2 \mathrm{~mm})$ and in the No Target $(205.3 \pm 4.2 \mathrm{~mm})$ than in the Target condition $(213.9 \pm 4.1 \mathrm{~mm})$. Interestingly, the VIBRATION by VISION interaction effect (see Figure $\underline{3 \mathrm{~A} Z})$ revealed that the effect of vibration was greater in the fixation condition $(38.7 \mathrm{~mm}$ amplitude reduction) than in the saccade condition (30.9 $\mathrm{mm}$ reduction). And finally, the interaction between VISION and TARGET revealed that the hand was undershooting the target more in the SAC condition, in particular when the targets were removed (SAC-NT $=197.6 \pm$ $3.6 \mathrm{~mm} ; \mathrm{SAC}-\mathrm{T}=213 \pm 3.6 \mathrm{~mm} ;$ FIX-NT $=213 \pm 5.3 \mathrm{~mm} ;$ FIX-T $=214.8 \pm 5.4 \mathrm{~mm}$ ).

Peak velocity $(P V)$. Significant main effects were found for VIBRATION $[F(1,19)=$ 61.964, $p<.001]$, and for TARGET $[F(1,19)=18.222, p<.001]$, and interaction effects involving VIBRATION and VISION $[F(1,19)=6.708, p<.05]$, and VIBRATION and TARGET $[F(1,19)=7.634, p<.05]$. In the condition without vibration, PV was higher $(758 \pm$ $27.1 \mathrm{~mm} / \mathrm{s})$ than when vibration was applied $(668 \pm 26.2 \mathrm{~mm} / \mathrm{s})$. Peak velocity was lower when there were targets presented $(701 \pm 25 \mathrm{~mm} / \mathrm{s})$ than when they were removed $(725.1 \pm 27.3$ $\mathrm{mm} / \mathrm{s}$ ). The interaction effects showed that the effect of vibration on PV was larger in the fixation versus the saccade condition $(\mathrm{SAC}-\mathrm{NoVib}=742.4 \pm 23.6 \mathrm{~mm} / \mathrm{s} ; \mathrm{SAC}-\mathrm{Vib}=664.4 \pm$ $23.87 \mathrm{~mm} / \mathrm{s} ;$ FIX-NoVib $=773.6 \pm 31.5 \mathrm{~mm} / \mathrm{s} ;$ FIX-Vib $=671.7 \pm 30.1 \mathrm{~mm} / \mathrm{s}) \underline{(\text { see Figure } 3 \mathrm{C})}$ and in the condition without targets compared to when targets were presented (NoTAR-NoVib $=751.2 \pm 26 \mathrm{~mm} / \mathrm{s} ;$ NoTAR-Vib $=650.8 \pm 25.1 \mathrm{~mm} / \mathrm{s} ;$ TAR-NoVib $=764.8 \pm 28.7 \mathrm{~mm} / \mathrm{s} ;$ TAR-Vib $=685.3 \pm 27.5 \mathrm{~mm} / \mathrm{s})$.

Time to peak velocity (\%TTPV). Only a main effect of VISION was found for \%TTPV $[F(1,19)=4.813, p<.05]$. In the eye-hand coordinated condition (SAC), the time that the hand needed to reach peak velocity was shorter $(36.8 \pm 1.4 \%$ of the total hand movement time) than in the eye fixation condition $(37.2 \pm 1.4 \%)$. 


\subsection{Eye movements and eye-hand coordination}

In Figure $4 \underline{3}$, an exemplar trial is shown with the eye and hand position plotted over time in a vibration condition without targets.

Number of eye movements. The ANOVA revealed a main effect for TARGET $[F(1,19)$ $=6.313, p<.05]$. Significantly more hand movements were accompanied by saccades in the target condition $(83.9 \pm 1.9 \%)$ than when no targets were presented $(80.5 \pm 1.6 \%)$.

Amplitude of the primary saccade (AMPs). The analysis revealed significant main effects for VIBRATION $[F(1,19)=7.030, p<.05]$ and for TARGET $[F(1,19)=6.348, p<.05]$. 
Interestingly, the amplitude of the primary saccade was shortened by $3.6 \%$ in the condition when local tendon vibration was applied to the hand movement (no vibration: $197.8 \pm 4.6 \mathrm{~mm}$; vibration: $190.8 \pm 5.5 \mathrm{~mm}$ ). The eye movement was also shorter in the target condition (189.2 $\pm 6.1 \mathrm{~mm}$ ) than when the targets were removed $(199.4 \pm 4.3 \mathrm{~mm})$.

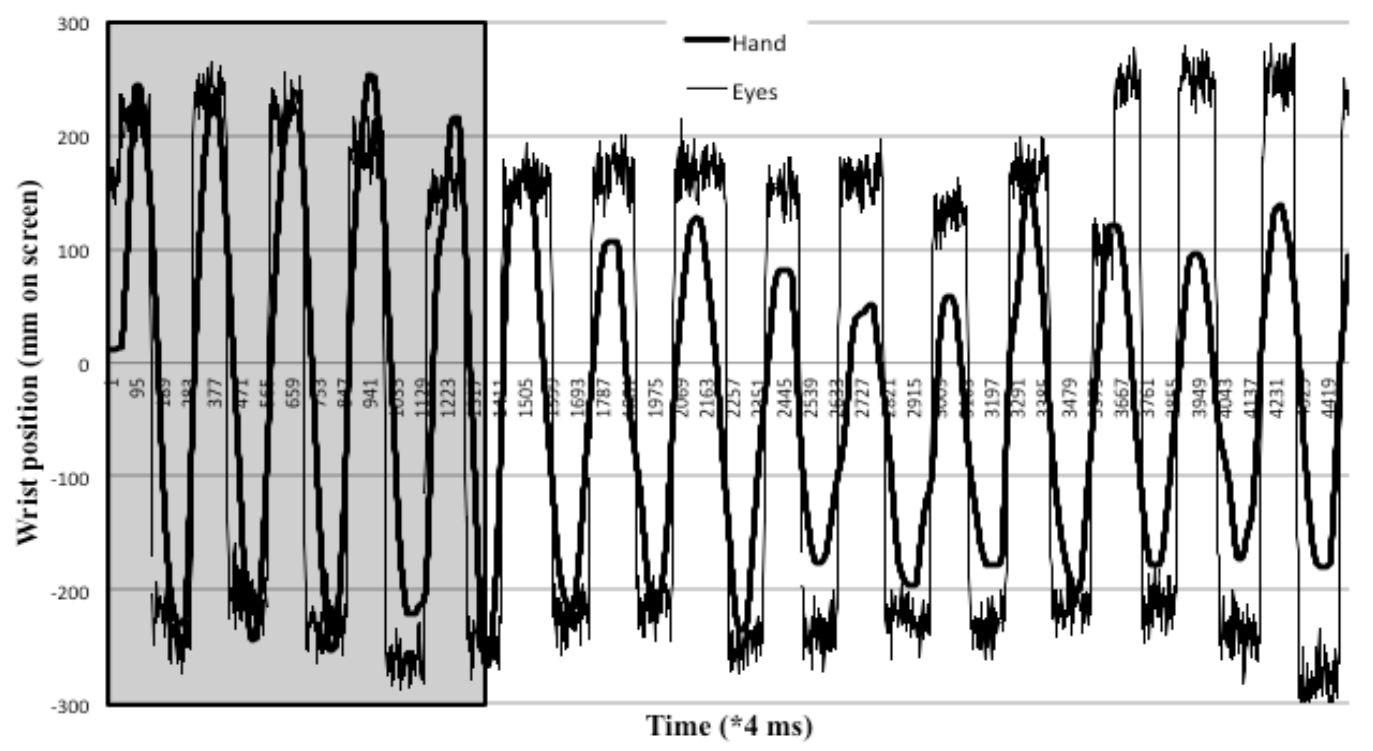

Figure 43: Instance of trial showing the effect of tendon vibration on hand and eye movements. Both cursor and targets disappeared after the $10^{\text {th }}$ beep (gray area). Vibrating the wrist extensors caused a decrease in amplitude of eye (primary saccade) and hand movements.

Eye-hand coordination. There was no influence of tendon vibration nor vision on the relative timing of eye and hand movements. On average, the primary saccade started when the hand had passed $10.9 \pm 2.4 \%$ of its movement time and ended at $20.9 \pm 2.4 \%$ of hand movement time. However, significant main effects for TARGET were found for both the start $[F(1,19)=9.715, p<.01]$ and end of the primary saccade $[F(1,19)=7.03, p=0.016]$. The primary saccade started and ended earlier in the condition without targets (SSAC: NoTAR $=18.5 \pm$ $2.7 \% ; \mathrm{TAR}=13 \pm 2.9 \% ; \mathrm{ESAC}: \mathrm{NoTAR}=18.9 \pm 2.6 \% ; \mathrm{TAR}=23.5 \pm 2.7 \%)$.

\section{DISCUSSION}

Even though reciprocal or cyclical aiming movements are not just a concatenation of discrete movements (Terrier et al., 2011), laws and relationships are usually consistent over the two types of task (e.g., Binsted \& Elliott, 1999; Fitts \& Peterson, 1964; Fitts, 1954; Lavrysen 
et al., 2006, Van Halewyck, Lavrysen, Levin, Elliott \& Helsen, 2014). Moreover, cyclical aiming paradigms can provide specific methodological advantages. For example, with tendon vibration (TV) it takes time for the shaker to reach stable amplitude and therefore the effect to be maximally observed. Furthermore, a transfer between eye and hand feedforward and feedback signals is less likely in discrete aiming tasks as the eye movement typically precedes the hand movement. Because of the succession of movements, a cyclical aiming paradigm optimizes the expression of illusory bias and allows for an interplay between sensory information across consecutive movements. Furthermore, controlling movement speed through the use of a metronome allows examining aiming error uncontaminated by the typical speedaccuracy trade-offs seen in discrete aiming.

The goal of the present experiment was to investigate the impact of visual and proprioception sensory information on goal-directed eye and hand movements. Three main issues were investigated: 1) the effect of local muscle TV on eye and hand movements; 2) the impact of visual information on this vibration effect; 3) the impact of vibration on eye-hand coordination. This study also adds to our more general understanding of illusions and perceptual-motor control by using a proprioceptive illusion to build on the vast literature addressing the impact of visual illusions on limb control.

With respect to our first research goal, tendon vibration of the wrist extensor muscles caused the hand to undershoot the target (Cody, Schwartz, \& Smit, 1990; Kasai et al., 1992, 1994; Verschueren et al., 1998). This reduction in amplitude was more than $15 \%$ of the total amplitude in the unbiased condition. As all trials were performed without cursor vision, participants did not notice that they were making smaller wrist movements. The illusory decrease in amplitude coincided with a decrease in peak velocity. Hence, participants executed shorter movements with lower peak velocity when vibration was given while maintaining movement time. Surprisingly, the primary saccades were also shorter in the trials with vibration, but to a lesser extent than wrist movements (3.6\% reduction of amplitude). As saccades typically precede hand movements, the exchange of a priori target information is unlikely here because the participants had no notion that their hand did not make the full range of movement. Prior knowledge is a prerequisite for predictive information exchange from hand to eye movements (van Donkelaar et al., 2004). Thus, the differences observed here cannot be explained by an efference-copy signal based on prior knowledge of the movement to be performed. Alternatively, as suggested by van Donkelaar et al. (2004), the efference copy of the movement kinematics might have interacted with the saccadic planning process. Although we did not directly measure forces, both the amplitude and peak velocity were lower in the 
vibration trials. Also in the tendon vibration experiment by Tardy-Gervet and colleagues (1989), illusory gaze displacements were induced by locally vibrating the arm when explicitly instructing the participants to look at the position of the hand as they felt it was moving. As in our study, participants were not aware, and therefore did not plan the shorter hand movements in advance. Therefore, we propose that in addition to anticipatory control, proprioceptive feedback also contributed to the observed effects on saccades. Apart from a general influence of hand movements on saccades, perceptual illusions have been shown to influence eye movements in different domains. Visual (ML illusion), proprioceptive (TV; e.g., Tardy-Gervet et al., 1989), or even auditory perturbations (e.g., Fracasso, Targher, Zampini, \& Melcher, 2013) influence gaze. Regarding TV, the present study is, to our knowledge, the first to demonstrate an effect of local arm vibration on saccades in a dynamic eye-hand coordination task.

As an explanation for these results, van Donkelaar and colleagues (2004) described a processing scheme with an interaction between eye and hand target representations. This model shows the interaction between the hand and eye movements in a single movement cycle. As saccades occur earlier in the movement cycle than hand movements, the illusion effect on the preceding hand movement may influence the planning of the next saccade. In this way, erroneous feedback from one movement could have led to an update and shift in the target representation used for both eye and hand movements (Sailer, Eggert, Ditterich, \& Straube, 2002). Based on the discrepancy between the planned (efference copy) and perceived wrist position after the first movement with TV, an erroneous efference copy associated with the next wrist movement might have influenced the planning of the subsequent eye movement. An exchange of information between the reach plan and the saccadic plan is therefore being proposed to corroborate the mutual interaction between saccadic and reach plans, and between subsequent movements. Recently, the Frontal Eye Fields, a brain region located in the frontal cortex, has been suggested as possible candidate for the integration of signals from the hand motor system in the planning and execution of eye movements (Thura, Hadj-Bouziane, Meunier, \& Boussaoud, 2008).

The differential effects of TV on hand and eye movements seem to be at odds with visual (ML) illusion studies in which typically greater and more consistent bias is found for saccades than hand movements. The effect on saccades was probably reduced here because the target location was not biased (as in visual illusions). Therefore, the saccades could be programmed based on accurate information and were only slightly affected by the changed target representation due to TV. Interestingly, the presence (or absence) of targets did not 
increase (decrease) this interference, contrary to ML studies in which misperceptions of target representations typically increase when dependent on memory (Lavrysen et al., 2006). Although the expression and intensity of the bias is not the same, both ML and TV studies have often shown effects on both effectors, highlighting yet again the cross-talk and mutual influences between eye and hand movement systems (Gauthier \& Mussa Ivaldi, 1988). Other evidence of this mutual interaction might be provided by the interaction effects of vision $x$ vibration and vision $\times$ target on hand amplitude. The reduced saccades might have influenced the hand amplitude and caused or augmented the consistent target undershoot, even in the absence of vibration.

Secondly, the impact of (the availability of) visual information on the magnitude of the illusion was examined. All trials were performed without retinal feedback of hand movement, as seeing the hand position might have caused an updating and correcting of the movement plans of both eyes and hand (Elliott et al., 2010). The availability and quality of retinal (target) and extraretinal (saccade) information about the distance to be travelled was manipulated. In the absence of vision of the hand and target, the participant could only address previously available knowledge and current proprioceptive information. Moreover, by perturbing proprioception, the participant was forced to use any available extraretinal and target information to plan and update the movement plan. As the accuracy of the movements is dependent on the vision condition, the no-vibration conditions for each of the vision conditions were the baseline for the corresponding vibration trials. This enabled us to compare performance and illusion effects under similar vision conditions.

The results showed that moving the hand while making saccades diminished the illusion effect induced by the proprioceptive bias. This effect was manifested by smaller differences in hand amplitude between vibration and no vibration conditions when saccades were allowed as compared to observed amplitude differences in the fixation condition. When making saccades in the absence of retinal (target) information, participants may have been more dependent on extraretinal information to improve movement accuracy (Binsted \& Elliott, 1999), which could have helped diminishing the effect of the illusion. In the saccade condition, participants may have made more use of their unperturbed extraretinal information to perform the task, because they could not rely on accurate proprioceptive information (Bekkering \& Sailer, 2002; Binsted $\&$ Elliott, 1999). In this theoretical context, it has also been shown that the importance of vision for postural stability increases when the proprioception is disturbed (De Nunzio, Nardone, \& Schieppati, 2005). Interestingly, tendon vibration had less impact on hand movements when they were combined with eye movements than when they were performed in isolation. This 
occurred despite biased saccades in the vibration condition. The presence of targets did not impact the vibration effect. This result further demonstrates some exchange of information between target representations for eye and for hand movements, and the flexibility of the visuomanual control system in sensory (re)weighting based on the task demands and information available. Here, this may involve some sort of averaging or regression toward the mean movement representation associated with the two systems.

Similar to visual perturbation paradigms (Lavrysen, Elliott, Buekers, Feys, \& Helsen, 2007), visual information impacted the magnitude of the tendon vibration effect. However, the type of information mediating this effect was different. In visual illusions, co-occurrence of saccades is a prerequisite for the expression of limb bias, whereas in our proprioceptive illusion paradigm, the saccades tended to have a beneficial mediating effect. In ML illusions, the perceptual bias is enhanced by making saccades and therefore having retinal visual information about the illusory percept. In contrast, in the TV paradigm, more accurate target information can be obtained by making saccades to unperturbed targets. This might provide the basis for the beneficial effects of saccades on hand movements. Further evidence for this type of interaction comes from monkey (Gauthier \& Mussa Ivaldi, 1988) and human target tracking studies (Lavrysen et al., 2008). Eye and hand effectors have been shown to perform better in coordination than in isolation. This could be because coordinated, compared to isolated movements, induces the activation of a complementary 'coordinator control center' (Gauthier et al., 1988). This notion is consistent with the results of imaging studies showing additional activation for coordinated versus isolated movements (Lavrysen et al., 2008).

Our third major finding was that perturbed proprioception had little no impact on eyehand coordination. Interestingly, whereas tendon vibration shortened and slowed down the movement of the hand, the temporal pattern and expression of kinematic markers remained the same as shown by the time to peak velocity. The consistency in the coordination between eye and hand supports the notion that eye movements are performed to assist the hand in the final phase of its movement (Starkes, Helsen, \& Elliott, 2002) as well as in the planning of the next movement (Tipper et al., 2001). The eyes started to move after the hand had already travelled about $11 \%$ of the movement time, and the eyes arrived on the target at $21 \%$ of the hand movement time. This was before hand peak velocity that occurred at $37.5 \%$ of the movement time. This finding is consistent with numerous studies on eye-hand coordination, showing that the temporal coordination is optimized to ensure critical visual pickup (see Starkes et al., 2002 and Bekkering \& Sailer, 2002 for reviews). The tight temporal coupling between eye and hand 
movements is ideal to provide the hand with visual, proprioceptive and extraretinal information about the relative position of hand and target (Binsted et al., 2001; Starkes et al., 2002).

To conclude, the goal of the present study was to investigate the role of retinal and extraretinal information in a proprioceptive illusion paradigm. The combination of tendon vibration and vision manipulation provided an ideal tool for studying the relationship between proprioception and vision in manual aiming. This study provides clear evidence for strong interactions between hand and eye movements. Local tendon vibration affected arm movement amplitude, but also, to a smaller extent, saccade amplitude. The intensity of the illusion was reduced when participants were allowed to make saccades, thereby emphasizing the importance of extraretinal visual information. Eye-hand coupling was not affected by tendon vibration. These results highlight the strong and mutual interactions between eye and hand control.-Future $\underline{\text { studies in our lab involve applying this paradigm in manual asymmetries context (testing both }}$ hands of left- and right-handers) and aging. Herewith we hope to shed more light on the $\underline{\text { mechanisms involved and stability of the processes over time. }}$ 
The authors wish to thank Ir. Marc Beirinckx and Ir. Paul Meugens for providing invaluable guidance in designing the research equipment and the electronics. MPB is supported by the Research Foundation - Flanders (FWO). Requests for reprints should be sent to Dr.

540 Werner Helsen, Research Centre for Movement Control and Neuroplasticity, KU Leuven, 541 Tervuursevest 101, 3001 Leuven, Belgium. E-mail: werner.helsen@ kuleuven.be. 
1. The ANOVA on the Number of hand movements revealed a significant main effect 547 for VIBRATION $[F(1,19)=9.756, p<.01]$. Significantly fewer eye-hand coordinated 548 movements were made when vibration was applied (14.9 \pm 0.08$)$ than without vibration (15.1 $549 \pm 0.1)$.

550 
Ariff, G., Donchin, O., Nanayakkara, T., \& Shadmehr, R. (2002). A real-time state predictor in motor control: study of saccadic eye movements during unseen reaching movements. The Journal of Neuroscience, 22, 7721-7729. doi:22/17/7721 [pii]

Assländer, L., \& Peterka, R. J. (2014). Sensory reweighting dynamics in human postural control. Journal of Neurophysiology, 111, 1852-1864. doi:10.1152/jn.00669.2013

Bekkering, H., \& Sailer, U. (2002). Commentary: Coordination of eye and hand in time and space. In Progress in Brain Research (Vol. 140, pp. 365-373). doi:10.1016/S00796123(02)40063-5

Binsted, G., Chua, R., Helsen, W., \& Elliott, D. (2001). Eye-hand coordination in goaldirected aiming. Human Movement Science, 20, 563-585. doi:10.1016/S01679457(01)00068-9

Binsted, G., \& Elliott, D. (1999). Ocular perturbations and retinal/extraretinal information: the coordination of saccadic and manual movements. Experimental Brain Research, 127, 193-206. doi:10.1007/s002210050789

Binsted, G., \& Elliott, D. (1999). The Müller-Lyer illusion as a perturbation to the saccadic system. Human Movement Science, 18, 103-117. doi:10.1016/S0167-9457(98)00038-4

Bruno, N., Bernardis, P., \& Gentilucci, M. (2008). Visually guided pointing, the Müller-Lyer illusion, and the functional interpretation of the dorsal-ventral split: conclusions from 33 independent studies. Neuroscience and Biobehavioral Reviews, 32(3), 423-437. doi:10.1016/j.neubiorev.2007.08.006

Bruno, N., Knox, P. C., \& de Grave, D. D. J. (2010). A metanalysis of the effect of the Müller-Lyer illusion on saccadic eye movements: no general support for a dissociation of perception and oculomotor action. Vision Research, 50(24), 2671-2682. doi:10.1016/j.visres.2010.09.016

Casini, L., Romaiguère, P., Ducorps, A., Schwartz, D., Anton, J., \& Roll, J. (2011). Cortical correlates of illusory hand movement perception in humans: a MEG study. Brain Research, 1121, 200-206. doi: 10.1016/j.brainres.2006.08.124

Cignetti, F., Vaugoyeau, M., Nazarian, B., Roth, M., Anton, J-L., \& Assaiante, C. (2014). Boosted activation of right inferior frontoparietal network: A basis for illusory movement awareness. Human Brain Mapping, 35, 5166-5178. doi: 10.1002/hbm.22541

Cody, F. W., Schwartz, M. P., \& Smit, G. P. (1990). Proprioceptive guidance of human voluntary wrist movements studied using muscle vibration. The Journal of Physiology, 427, 455-470. doi:10.1016/0022-460X(91)90468-Y

Cordo, P., Gurfinkel, V. S., Bevan, L., \& Kerr, G. K. (1995). Proprioceptive consequences of tendon vibration during movement. Journal of Neurophysiology, 74, 1675-1688. 
De Nunzio, A. M., Nardone, A., \& Schieppati, M. (2005). Head stabilization on a continuously oscillating platform: The effect of a proprioceptive disturbance on the balancing strategy. Experimental Brain Research, 165, 261-272. doi:10.1007/s00221005-2297-7

Elliott, D., Hansen, S., Grierson, L. E. M., Lyons, J., Bennett, S. J., \& Hayes, S. J. (2010). Goal-directed aiming: two components but multiple processes. Psychological Bulletin, 136(6), 1023-1044. doi:10.1037/a0020958

Elliott, D., \& Lee, T. D. (1995). The role of target information on manual-aiming bias. Psychological Research, 58, 2-9. doi:10.1007/BF00447084

Fitts, P. M. (1954). The information capacity of the human motor system in controlling the amplitude of movement. 1954. Journal of Experimental Psychology. General, 47(6), 381-391. doi:10.1037/h0055392

Fitts, P. M., \& Peterson, J. R. (1964). Information capacity of discrete motor responses. Journal of Experimental Psychology, 67, 103-112. doi:10.1037/h0045689

Fracasso, A., Targher, S., Zampini, M., \& Melcher, D. (2013). Fooling the eyes: the influence of a sound-induced visual motion illusion on eye movements. PLOS ONE, 8 . doi:10.1371/journal.pone.0062131

Franz, V. H., \& Gegenfurtner, K. R. (2008). Grasping visual illusions: consistent data and no dissociation. Cognitive Neuropsychology, 25, 920-950. doi:10.1080/02643290701862449

Franz, V. H., Gegenfurtner, K. R., Bülthoff, H. H., \& Fahle, M. (2000). Grasping visual illusions: no evidence for a dissociation between perception and action. Psychological Science, 11, 20-25. doi:10.1111/1467-9280.00209

Gauthier, G. M., \& Mussa Ivaldi, F. (1988). Oculo-manual tracking of visual targets in monkey: role of the arm afferent information in the control of arm and eye movements. Experimental Brain Research, 73, 138-154. doi:10.1007/BF00279668

Gauthier, G. M., Vercher, J. L., Mussa Ivaldi, F., \& Marchetti, E. (1988). Oculo-manual tracking of visual targets: control learning, coordination control and coordination model. Experimental Brain Research, 73, 127-137. doi:10.1007/BF00279667

Gentilucci, M., Chieffi, S., Daprati, E., Saetti, M. C., \& Toni, I. (1996). Visual illusion and action. Neuropsychologia, 34, 369-376. doi:10.1016/0028-3932(95)00128-X

Goble, D.J., Coxon, J.P., Van Impe, A., Geurts, M., Doumas, M., Wenderoth, N., \& Swinnen, S. (2011) Brain activity during ankle proprioceptive stimulation predicts balance performance in young and older adults. The Journal of Neuroscience, 31, 16344-16352. doi: 10.1523/JNEUROSCI.4159-11.2011

Kasai, T., Kawanishi, M., \& Yahagi, S. (1994). Effects of upper limb muscle vibration on human voluntary wrist flexion-extension movements. Perceptual and Motor Skills, 78, 43-47. doi:10.2466/pms.1994.78.1.43 
Lavrysen, A., Elliott, D., Buekers, M. J., Feys, P., \& Helsen, W. F. (2007). Eye-hand coordination asymmetries in manual aiming. Journal of Motor Behavior, 39(1), 9-18. doi:10.3200/JMBR.39.1.9-18

Lavrysen, A., Helsen, W. F., Elliott, D., Buekers, M. J., Feys, P., \& Heremans, E. (2006). The type of visual information mediates eye and hand movement bias when aiming to a Müller-Lyer illusion. Experimental Brain Research, 174(3), 544-554. doi:10.1007/s00221-006-0484-9

Lavrysen, A., Heremans, E., Peeters, R., Wenderoth, N., Helsen, W. F., Feys, P., \& Swinnen, S. P. (2008). Hemispheric asymmetries in eye-hand coordination. NeuroImage, 39(4), 1938-1949. doi:10.1016/j.neuroimage.2007.10.007

Lazzari, S., Vercher, J. L., \& Buizza, A. (1997). Manuo-ocular coordination in target tracking. I. A model simulating human performance. Biological Cybernetics, 77, 257-266. doi: $10.1007 / \mathrm{s} 004220050386$

Lunenburger, L., Kutz, D. F., \& Hoffmann, K. P. (2000). Influence of arm movements on saccades in humans. European Journal of Neuroscience, 12, 4107-4116. doi:10.1046/j.1460-9568.2000.00298.x

Mendoza, J.E., Elliott, D., Meegan, D.V., Lyons, J.L. \& Welsh, T.N. (2006). The effect of the Muller-Lyer illusion on the planning and control of manual aiming movements. Journal of Experimental Psychology: Human Perception and Performance, 32, 413-422. doi:10.1037/0096-1523.32.2.413

Milner, A. D., \& Goodale, M. A. (2008). Two visual systems re-viewed. Neuropsychologia, 46, 774-785. doi:10.1016/j.neuropsychologia.2007.10.005

Naito, E. (2005). Sensing limb movements in the motor cortex: How humans sense limb movement. The Neuroscientist, 10, 73-82. doi: 10.1177/1073858403259628.

Romaiguère, P., Anton, J., Roth, M., Casini, L., \& Roll, J. (2003). Motor and parietal cortical areas both underlie kinaesthesia. Cognitive Brain Research, 16, 74-82. doi:10.1016/S0926-6410(02)00221-5

Sailer, U., Eggert, T., Ditterich, J., \& Straube, A. (2002). Global effect of a nearby distractor on targeting eye and hand movements. Journal of Experimental Psychology. Human Perception and Performance, 28, 1432-1446. doi:10.1037/0096-1523.28.6.1432

Starkes, J., Helsen, W., \& Elliott, D. (2002). A ménage à trois: the eye, the hand and on-line processing. Journal of Sports Sciences, 20, 217-224. doi:10.1080/026404102317284772

Steyvers, M., Verschueren, S.M.P., Levin, O., Ouamer, M., \& Swinnen S.P. (2001). Proprioceptive control of cyclical bimanual forearm movements across different movement frequencies as revealed by means of tendon vibration. Experimental Brain Research, 140, 326-334. doi:-10.1007/s002210100819 
Tardy-Gervet, M. F., Gilhodes, J. C., \& Roll, J. P. (1989). Induction of illusory limb movement as a means of studying sensorimotor interactions in the eye-arm system. Brain, Behavior and Evolution, 33, 165-170. doi:10.1159/000115922

Terrier, R., Forestier, N., Berrigan, F., Germain-Robitaille, M., Lavallière, M., \& Teasdale, N. (2011). Effect of terminal accuracy requirements on temporal gaze-hand coordination during fast discrete and reciprocal pointings. Journal of Neuroengineering and Rehabilitation, 8(1), 10. doi:10.1186/1743-0003-8-10

Thura, D., Hadj-Bouziane, F., Meunier, M., \& Boussaoud, D. (2008). Hand position modulates saccadic activity in the frontal eye field. Behavioural Brain Research, 186(1), 148-153. doi:10.1016/j.bbr.2007.07.035

Tipper, S. P., Howard, L. a., \& Paul, M. a. (2001). Reaching affects saccade trajectories. Experimental Brain Research, 136(2), 241-249. doi:10.1007/s002210000577

Van Donkelaar, P. (1999). Pointing movements are affected by size-contrast illusions. Experimental Brain Research, 125, 517-520. doi:10.1007/s002210050710

Van Donkelaar, P., Siu, K.-C., \& Walterschied, J. (2004). Saccadic output is influenced by limb kinetics during eye-hand coordination. Journal of Motor Behavior, 36(3), 245-252. doi:10.3200/JMBR.36.3.245-252

Van Halewyck, F. Lavrysen, A., Levin, O., Boisgontier, M. P., Elliott, D., \& Helsen, W. F. (2014). Both age and physical activity level impact on eye-hand coordination. Human Movement Science, 36, 80-96. doi:10.1016/j.humov.2014.05.005

Van Halewyck, F., Lavrysen, A., Levin, O., Elliott, D., \& Helsen, W.F. (2014). The impact of age and physical activity level on manual aiming performance. Journal of Aging and Physical Activity, 23(2), 169-179. doi: 10.1123/japa.2013-0104.

Vercher, J. L., Gauthier, G. M., Guédon, O., Blouin, J., Cole, J., \& Lamarre, Y. (1996). Selfmoved target eye tracking in control and deafferented subjects: roles of arm motor command and proprioception in arm-eye coordination. Journal of Neurophysiology, 76(2), 1133-1144. Retrieved from http://www.ncbi.nlm.nih.gov/pubmed/8871226

Verschueren, S. M., Cordo, P. J., \& Swinnen, S. P. (1998). Representation of wrist joint kinematics by the ensemble of muscle spindles from synergistic muscles. Journal of Neurophysiology, 79, 2265-2276. 